\title{
A Numerical Adaptive Algorithm for the Obstacle Problem
}

\author{
F.A. Pérez, J.M. Cascón, and L. Ferragut \\ Departamento de Matemática Aplicada, Universidad de Salmanca, \\ 37008 Salamanca, Spain \\ \{franc, casbar, ferragut\}@usal.es \\ http://matapli.fis.usal.es
}

\begin{abstract}
This paper concerns an adaptive finite element method for the elliptic obstacle problem. We consider the formulation of the problem as an elliptic variational inequation. The adaptive algorithm (modified Uzawa adaptive method)we construct is based on a combination of the Uzawa method associated with the corresponding multivalued operator and a convergent adaptive method for the linear problem. As our main result we show that if the adaptive method for the linear problem is convergent, then the adaptive modified Uzawa method is convergent as well. A numerical experiment shows the studied properties of the method.
\end{abstract}

\section{Introduction}

Adaptive mesh refinement based on a posteriori error estimates is an essential instrument for efficient numerical solving of Partial Differential Equations, in particular nonlinear problems.

The obstacle problem may be considered as a model problem for variational inequalities (see, e.g, [1], 2], [5], 6], [7] ). The obstacle problem belongs to a class of problems which have found applications in such diverse fields as elasticity and fluid dynamics(see,e.g, 4], [5], [6], [7]). These problems are characterized by free boundaries that are a priori unknown. An issue to consider is that in their formulation the solution is sought in a convex and not necessarily linear subset of some vector space.

In this paper we construct an adaptive finite element method for the elliptic obstacle problem (adaptive modified Uzawa method). We formulate the variational inequality in terms of a multivalued operator. As it is well known, the Uzawa algorithm consists in solving in each iteration a linear problem and a nonlinear adaptation of the Lagrange multiplier associated with the multivalued equation. As our main result we show that if the adaptive method applied for the linear equation is convergent then, the adaptive modified Uzawa method is convergent as well.

The convergence is proved with respect to a discrete solution in the space corresponding to a sufficiently refined mesh. In order to assure the convergence of the Lagrange multiplier, the space of piecewise constant finite element functions is extended with bubble functions. 
We get the following convergence result:

Main result. Let $\left(U_{j}, P_{j}\right)$ the sequence of finite element solutions of the linear problem and the corresponding Lagrange multiplier produced by the adaptive modified Uzawa algorithm. There exist positive constants $C$ and $\delta<1$ such that

$$
\left\|u-U_{j}\right\|+\left|p-P_{j}\right| \leq C \delta^{j}
$$

where $|\cdot|$ is the $L^{2}(\Omega)$ norm and $\|\cdot\|:=|\nabla \cdot|$ is the energy norm; $u$ and $p$ are the discrete solutions on a sufficiently refined mesh.

Several examples show the convergence of the method. The numerical experiments have been developed with the finite element toolbox ALBERT [11, extended with new function basis including bubble functions.

\section{Continuous Problem}

Let $\Omega$ a bounded, polyhedral domain in $\mathbb{R}^{d}(d=2,3) \cdot L^{2}(\Omega)$ the space of Lebesgue-measurable functions that are square-integrable. Set $\mathbb{W}:=H^{1}(\Omega)$ the Sobolev space of functions in $L^{2}(\Omega)$ with weak derivatives of first order in $L^{2}(\Omega)$. $\mathbb{V}:=H_{0}^{1}(\Omega)$ is the subspace of $\mathbb{W}$ whose functions vanish on boundary $\partial \Omega$. In the following, $(\cdot, \cdot)$ denotes the inner product in $L^{2}$ and $\langle\cdot, \cdot\rangle$ the duality pairing between the corresponding spaces. Moreover, consider the bilinear and linear forms:

$$
\begin{array}{r}
a(u, v)=(\nabla u, \nabla v),<A u, v>=a(u, v), \forall u, v \in \mathbb{V} \\
<F, v>=(f, v)
\end{array}
$$

The rest of the data is given by a force density $f \in L^{2}(\Omega)$ and an obstacle $\psi \in \mathbb{W}$ with $\psi \leq 0$ on $\partial \Omega$. The obstacle $\psi$ is associated with the nonempty, $\mathbb{V}$-closed, and convex set of admisible displacements:

$$
\mathcal{K}:=\{v \in \mathbb{V} \mid v \geq \psi \text { a.e. in } \Omega\}
$$

The continuous problem reads as follows:

Continuous Problem. Given $\Omega, f, \psi$ as above, find $u \in \mathcal{K}$ such that the following variational inequality holds:

$$
a(u, v-u) \geq(f, v-u) \forall v \in \mathcal{K}
$$

It is well known that the above problem admits a unique solution $u$, see, e.g. [3]. The unilateral constraint $u \geq \psi$ yields a line singularity (free boundary) that is the internal boundary of the contact set:

$$
\mathcal{C}:=\{x \in \Omega: u(x)=\psi(x)\}
$$

The free boundary location is a priori unknown and a prime computational objective. 


\section{Formulation with Multivalued Operator}

Consider the functional on $\mathbb{V}$ that characterizes the convex $\mathcal{K}$ :

$$
I_{\mathcal{K}}(v)= \begin{cases}0 & v \in \mathcal{K} \\ \infty & v \notin \mathcal{K}\end{cases}
$$

The problem (4) is equivalent to: Find $u \in \mathbb{V}$ such that

$$
\begin{aligned}
& A u+p=F \text { in } \mathbb{V}^{\prime} \\
& p \in \partial I_{\mathcal{K}}(u) \text { in } \mathbb{V}^{\prime}
\end{aligned}
$$

where $\partial I_{\mathcal{K}}$ is the subdiferential of $I_{\mathcal{K}}$, which is a multivalued operator, and $\mathbb{V}^{\prime}$ is the dual space of $\mathbb{V}$. Set $\Pi_{\lambda}$ the Yosida approximation of $\partial I_{\mathcal{K}}$. The solution is then characterized by the existence of $p \in \mathbb{V}$, such that the pair $(u, p) \in \mathbb{V} \times \mathbb{V}$ holds:

$$
\begin{array}{r}
A u+p=F \text { in } \mathbb{V}^{\prime} \\
p=\Pi_{\lambda}(u+\lambda p) \text { in } \mathbb{V}^{\prime}
\end{array}
$$

The operator $\Pi_{\lambda}=\frac{1}{\lambda}\left(I d-P_{\mathcal{K}}\right)$, where $P_{\mathcal{K}}$ is the projection operator on $\mathcal{K}$. Moreover, $\Pi_{\lambda}$ is a Lipschitz operator with constant $1 / \lambda$

\section{Discretization}

Let $\mathcal{T}_{h}$ be a uniformly regular triangulation of $\Omega$ characterized by the diameter $h$. Let $\mathbb{V}_{h} \subset \mathbb{V}$ indicate the space of continuous piecewise linear finite element functions and extended with the bubble functions over $\mathcal{T}_{h}$, i.e., $\mathbb{V}_{h}:=\left\{v_{h} \in\right.$ $\left.\mathcal{C}^{0}(\bar{\Omega}) \mid \quad \forall T \in \mathcal{T}_{h}, \quad v_{h \mid T} \in P_{1}(T)+B(T)\right\}$, where $B(T)$ is generated by the product $\lambda_{1} \lambda_{2} \lambda_{3}$ of the barycentric coordinates. Let $\mathbb{M}_{h} \subset L^{2}(\Omega)$ be the space of piecewise constant finite element functions over $\mathcal{T}_{h}$.

We consider the operator $\Pi_{\lambda, h}=\frac{1}{\lambda}\left(I d-P_{\mathcal{K}_{h}}\right)$, where $\mathcal{K}_{h}$ is a suitable approximation of $\mathcal{K}$, that is, $\mathcal{K}_{h}=\left\{q \in \mathbb{M}_{h} \mid \quad q_{\mid T} \geq \psi\left(\mathfrak{b}_{T}\right) \quad \forall T \in \mathcal{T}_{h}\right\}$, being $\mathfrak{b}_{T}$ the barycentre of $T$. The discrete problem reads as follows:

Find $\left(u_{h}, p_{h}\right) \in \mathbb{V}_{h} \times \mathbb{M}_{h}$ such that

$$
\begin{aligned}
A u_{h}+R^{t} p_{h} & =F \\
p_{h} & =\Pi_{\lambda, h}\left(R u_{h}+\lambda p_{h}\right)
\end{aligned}
$$

where $R$ denotes the orthogonal projection operator in $L^{2}(\Omega)$ norm from $\mathbb{V}_{h}$ onto $\mathbb{M}_{h}$ and $R^{t}$ is its transposed operator.

The Uzawa algorithm iterations are written:

For any $p_{h}{ }^{(0)}$, obtained $p_{h}^{(i-1)}$

$$
\begin{aligned}
A u_{h}{ }^{(i)} & =F-R^{t} p_{h}{ }^{(i-1)} \\
p_{h}{ }^{(i)} & =\Pi_{\lambda, h}\left(R u_{h}{ }^{(i)}+\lambda p_{h}{ }^{(i-1)}\right)
\end{aligned}
$$


It is well known that the above algorithm is convergent: for some $\lambda>0$ we have

$$
\lim _{i \rightarrow \infty}\left\|u_{h}^{(i)}-u_{h}\right\|=0
$$

Remark 1. Notice that the above algorithm may be considered as a fixed point iteration for the application defined over $M_{h}$ :

$$
q \rightarrow \Pi_{\lambda}\left(\lambda\left(I-\frac{1}{\lambda} R A^{-1} R^{t}\right) q+R A^{-1} F\right)
$$

Since $\mathbb{V}_{h}$ and $\mathbb{M}_{h}$ are finite dimensional spaces and $\mathbb{V}_{h}$ has bubble functions, the kernel of $R^{t}$ is null. Thus, the application

$$
\begin{aligned}
\mathbb{M}_{h} & \rightarrow \mathbb{R} \\
q & \rightarrow\left|R^{t} q\right|
\end{aligned}
$$

defines a norm in $M_{h}$. We can now choose $\lambda$ such that:

$$
\left|I-\frac{1}{\lambda} R A^{-1} R^{t}\right|=1-\beta(h)<1
$$

hence,

$$
\left|p_{h}{ }^{i}-p_{h}\right| \leq(1-\beta(h))\left|p_{h}^{i-1}-p_{h}\right|
$$

and

$$
\lim _{h \rightarrow 0} \beta(h)=0
$$

\section{Adaptive Algorithm}

In this section we describe the adaptive-modified Uzawa method. To simplify notations let us assume that $\mathcal{T}_{j}$ stands for the mesh obtained from $\mathcal{T}_{j-1}$ by refining and the corresponding sets of finite element functions are denoted by $\left(\mathbb{V}_{j}, \mathbb{M}_{j}\right)$ and $\left.\left(\mathbb{V}_{j-1}, \mathbb{M}_{j-1}\right)\right)$. Consider a pair of successions:

$$
\begin{aligned}
\mathbb{V}_{0} \subset \mathbb{V}_{1} \subset \ldots \subset \mathbb{V}_{j} \subset \mathbb{V}_{j+1} \subset \ldots \subset \mathbb{V}_{J}=\mathbb{V}_{h} \\
\mathbb{M}_{0} \subset \mathbb{M}_{1} \subset \ldots \subset \mathbb{M}_{j} \subset \mathbb{M}_{j+1} \subset \ldots \subset \mathbb{M}_{J}=\mathbb{M}_{h}
\end{aligned}
$$

For any $P_{0} \in \mathbb{M}_{0}, \varepsilon_{0}>0,0<\gamma<1$. Given $P_{j-1} \in \mathbb{M}_{j-1}$, let $u_{j} \in V_{J}$ denote the solution of

$$
a\left(u_{j}, v\right)=<F, v>-\left(P_{j-1}, v\right) \quad \forall v \in \mathbb{V}_{J}
$$

An adaptive FEM method is applied to find $U_{j} \in V_{j}$ such that

$$
\left\|U_{j}-u_{j}\right\| \leq C \varepsilon_{j}, \quad\left|\left(R_{j}-R_{J}\right) U_{j}\right|<C \varepsilon_{j}
$$

where $\varepsilon_{j}<\gamma \varepsilon_{j-1}$. This procedure is denoted by 


$$
\left(\mathcal{T}_{j}, U_{j}\right) \leftarrow \operatorname{ELLIPTIC}\left(\mathcal{T}_{j-1}, U_{j-1}, \varepsilon_{j}, f\right)
$$

We, finally, actualize the multiplier:

$$
P_{j}=\Pi_{\lambda, j}\left(R_{j} U_{j}+\lambda P_{j-1}\right)
$$

The following box describes the algorithm:

\section{Adaptive Modified Uzawa Algorithm AMUA.}

Choose parameters $\lambda>0,0<\gamma<1, \varepsilon_{0}>0$; set $j=1$.

1. Select any initial mesh $\mathcal{T}_{0}$ and any $P_{0} \in \mathbb{M}_{0}$.

2. Update $\varepsilon_{j} \leftarrow \gamma \varepsilon_{j-1}$.

3. Compute $\left(\mathcal{T}_{j}, U_{j}\right) \leftarrow \operatorname{ELLIPTIC}\left(\mathcal{T}_{j-1}, U_{j-1}, P_{j-1}, \varepsilon_{j}, f\right)$.

4. Compute $P_{j} \leftarrow \operatorname{UPDATE}\left(\mathcal{T}_{j}, U_{j-1}, P_{j-1}, \lambda\right)$.

5. Update $j \leftarrow j+1$.

6. Go to step 2 .

With the hypothesis above, we have the following convergence theorem for the algorithm:

Theorem 1. There exist positive constants $C$ and $\delta<1$ such that the iterative solutions $\left(U_{j}, P_{j}\right)$ produced by the adaptive-modified Uzawa method satisfy:

$$
\left\|u_{J}-U_{j}\right\|+\left|p_{J}-P_{j}\right| \leq C \delta^{j}
$$

where $\left(u_{J}, p_{J}\right) \in \mathbb{V}_{J} \times \mathbb{M}_{J}$ denote the solution of the problem (11)-(12).

Sketch of the proof in the case $\psi=0$ :

$$
\begin{aligned}
P_{j} & =\Pi_{\lambda, j}\left(R_{j} U_{j}+\lambda P_{j-1}\right)= \\
& =\Pi_{\lambda, j}\left(R_{J} u_{j}+\lambda P_{j-1}+R_{J}\left(U_{j}-u_{j}\right)+\left(R_{j}-R_{J}\right) U_{j}\right)
\end{aligned}
$$

The solution $u_{j}$ of (20) may be written as $u_{j}=A^{-1}\left(F-R_{J}^{t} P_{j-1}\right)$. Observe that in the case $\psi=0$ we have $\Pi_{j}(z)=\Pi_{J}(z)$ if $z \in \mathbb{M}_{j}$. Hence

$$
P_{j}=\Pi_{\lambda, J}\left(\lambda\left(I-\frac{1}{\lambda} R_{J} A^{-1} R_{J}^{t}\right) P_{j-1}+R_{J} A^{-1} F+R_{J}\left(U_{j}-u_{j}\right)+\left(R_{j}-R_{J}\right) U_{j}\right)
$$

If we write $p_{J}$, the solution of (12), as follows

$$
p_{J}=\Pi_{\lambda, J}\left(\lambda\left(I-\frac{1}{\lambda} R_{J} A^{-1} R_{J}^{t}\right) p_{J}+R_{J} A^{-1} F\right)
$$

Then, subtracting (27) and (28), applying norms, we find an upper bound, for different constants $C$

$$
\begin{array}{r}
\left|p_{J}-P_{j}\right| \leq(1-\beta(h))\left|p_{J}-P_{j-1}\right|+\frac{C}{\lambda}\left\|U_{j}-u_{j}\right\|+\frac{C}{\lambda}\left|\left(R_{j}-R_{J}\right) U_{j}\right| \\
\left|p_{J}-P_{j}\right| \leq(1-\beta(h))\left|p_{J}-P_{j-1}\right|+\frac{C}{\lambda} \varepsilon_{0} \gamma^{j}
\end{array}
$$


As in [9], by induction arguments we obtain

$$
\left|p_{J}-P_{j}\right| \leq\left|p_{J}-P_{0}\right| \eta^{j}+\frac{1}{\lambda} \varepsilon_{0} j \eta^{j} \leq C \delta^{j}
$$

where $\eta=\max \{(1-\beta(h)), \gamma\}$ and $\eta<\delta<1$.

To find an error bound for $\left\|u_{J}-U_{j}\right\|$, observe that $\left\|u_{J}-u_{j}\right\| \leq C\left|p_{J}-P_{j}\right|$. Hence,

$$
\left\|u_{J}-U_{j}\right\| \leq\left\|u_{J}-u_{j}\right\|+\left\|u_{j}-U_{j}\right\| \leq C\left(\left|p_{J}-P_{j}\right|+\varepsilon_{j}\right)
$$

which proves the result.

For the case $\psi \neq 0$ we need to add $\left|\psi-\psi_{j}\right|<C \varepsilon_{j}$ to the condition (21) where $\psi_{j}$ is the interpolated function in $\mathbb{M}_{j}$.

\section{$6 \quad$ Numerical Experiment}

Consider $\Omega=\left\{x \in \mathbb{R}^{2}:\|x\|<1\right\}, f(x)=0$, and $\psi(x)=\max \{0,1-4\|x\|\}, \forall x \in$ $\Omega$. The solution of the problem $u(x)=1-\|x\|$, for all $x \in \Omega$ and $\mathcal{C}(u)=\{0\}$

Let us assume an initial triangulation $\mathcal{T}_{0}$ of $\Omega$, and the posteriori error estimate

$$
\left\|u-u_{h}\right\| \leq \eta\left(u_{h}\right)=\left(\sum_{T \in \mathcal{T}_{0}} \eta_{T}\left(u_{h}\right)^{2}\right)^{1 / 2}
$$

where(see [10] for $H^{1}$ error estimate for the linear problem $\left.-\Delta u=f(x)\right)$

$$
\begin{gathered}
\eta_{T}\left(u_{h}\right)^{2}=C_{0}^{2} h_{T}^{2}\left\|-\Delta u_{h}+f(\cdot)\right\|_{L^{2}(T)}^{2}+ \\
+C_{1}^{2} \sum_{\partial T \cap \Omega} h_{T}\left\|\llbracket \nu \cdot \nabla u_{h} \rrbracket\right\|_{L^{2}(\Gamma)}^{2}
\end{gathered}
$$

If $t o l$ is a given allowed tolerance for the error, and $\eta\left(u_{h}\right)>t o l$, we refine the mesh while $\eta>$ tol. For the Maximum strategy (see [11]), a threshold $\gamma \in(0,1)$ is given, and all elements $T \in \mathcal{T}_{j}$ with

$$
\eta_{T}>\gamma \max _{T^{\prime} \in \mathcal{T}} \eta_{T^{\prime}}
$$

are marked for refinement.

Set $\gamma=0.8, \varepsilon_{0}=$ tol $=1.0$ and the Yosida parameter $\lambda=1.0$. Figure [1] shows the behaviour of the true error in $H^{1}$-norm as a function of the number of $u_{h}$-degrees of freedom (DOF). We observe the improvement in applying our adaptive method (in solid linestyle) compared with the results obtained with uniform refinement (in dashed linestyle). Figure 2 shows mesh in the final step and the solution isolines. 


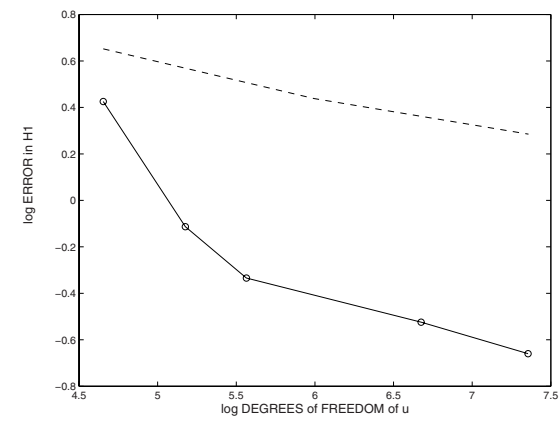

Fig. 1. $\log$-log error and DOF for $\varepsilon_{0}=1.0$
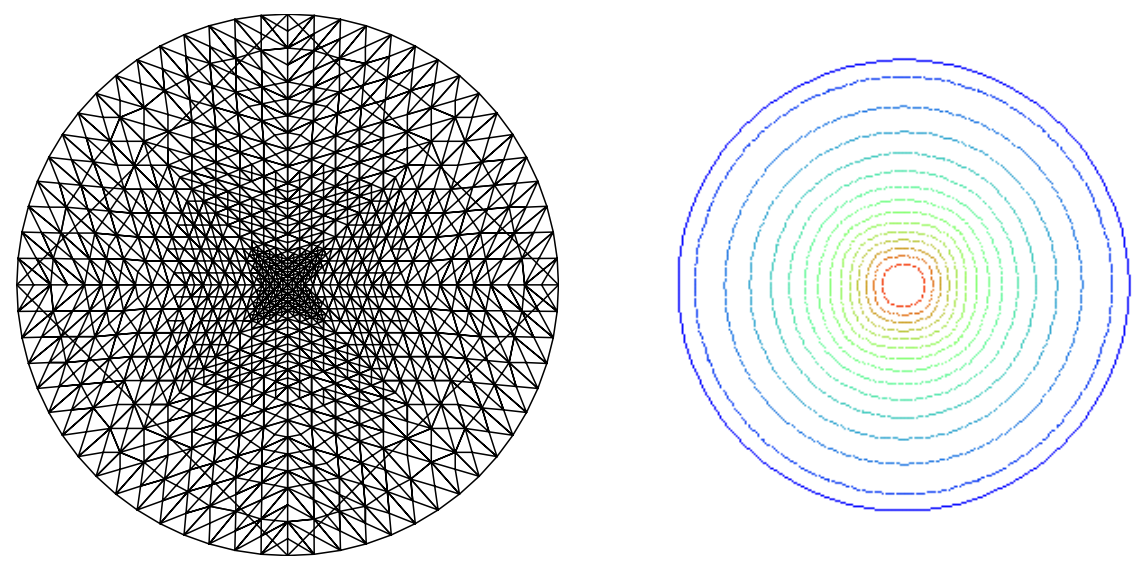

Fig. 2. Mesh and solution isolines

\section{Conclusions}

We have developed an adaptive Uzawa algorithm to solve the obstacle problem which is a modification of the classical Uzawa method. We justify the use of a a-posteriori error estimation from the linear elliptic problems for this kind of non-linear problems. The numerical results asserts the validity of the theoretical analysis and the efficiency of the algorithm. A better improvement should be obtained with a finest control of the interpolation error of the obstacle function $\psi$. This will be done in a future research.

Acknowledgements. Research partially supported by REN2001-0925-03-03, Ministerio de Ciencia y Tecnología (Spain) and SA089/01, Junta de Castilla y León (Spain). 


\section{References}

1. A. Friedman, Variational Principles and Free-Boundary Problems, Pure Appl. Math.,John Wiley, New York, 1982.

2. J. Haslinger, I. Hlaváček and J. Nečas, Numerical Methods for unilateral problems in solid mechanics, Handbook of Numerical Analysis. Vol. IV, P. G. Ciarlet and J. L. Lions, eds., North-Holland, Amsterdam, 1996, pp.313-485.

3. P. G. Ciarlet, Basic Error Estimates for Elliptic Problems, Handbook of Numerical Analysis. Vol II, P. G. Ciarlet and J. L. Lions, eds., North-Holland, Amsterdam, 1991,pp.24-25.

4. G. Duvaut and J.L. Lions, Inequalities in Mechanics and Physics, Grundlehren Mathematischen Wiss, Springer-Verlag, Berlin, Heidelberg, New York, 1976.

5. N. Kikuchi and J. T. Oden, Contact Problems in Elasticity: A Study of Variational Inequalities and Finite Element Methods, SIAM Stud. Appl.Math. 8,SIAM, Philadelphia, 1988.

6. D. Kinderlehrer and G. Stampacchia,An Introduction to Variational Inequalities and Their Applications, Pure Appl. Math. 88, Academic Press, New York, 1980.

7. J.F. Rodrigues, Obstacle Problems in Mathematical Physics, North-Holland Math. Stud. 134, North-Holland, Amsterdam, 1987.

8. J. L. Lions, Quelques méthodes de résolution de problèmes aux limites non linéaires, Dunod, Paris, 1969.

9. E. Bänsch, P.Morin and R.H. Nochetto, An adaptive Uzawa fem for the Stokes problem: Convergence without the inf-sup condition, SIAM J. Numer. Anal., 40 (2002), 1207-1229.

10. E. Bänsch, Local mesh refinement in 2 and 3 dimensions, Impact Comput.Sci.Engrg.,3(1991), 181-191.

11. A. Schmidt and K.G. Siebert, ALBERT: An adaptive hierarchical finite element toolbox, Preprint 06/2000, Freiburg (2000). 